\title{
Análisis de costo total de propiedad (TCO) en un proyecto/inversión TI para el modelo tradicional y su comparación con la implementación en nube pública (IAAS) para las empresas ecuatorianas
}

\section{Analysis of the total cost of ownership (TCO) of an IT project/investment for the traditional model and its comparison with the public cloud implementation (IAAS) for Ecuadorian enterprises}

\author{
Eduardo Xavier Chica Bermúdez \\ Facultad de Ingeniería en Electricidad y Computación, \\ Escuela Superior Politécnica del Litoral, Guayaquil, Ecuador \\ eduardochica@gmail.com \\ Orcid: 0000-0002-9673-3529
}

\begin{abstract}
Resumen- En Ecuador ya empezó el debate sobre si el modelo tradicional (on premise) representa una mayor inversión en la implementación de proyectos de Tecnologías de Información (TI) con relación a la nube pública. En el presente trabajo se pretende mostrar un análisis que los administradores del Gobierno TI deberían considerar a la hora de realizar la selección de su infraestructura. Se utiliza el modelo Costo Total de Propiedad (TCO), herramienta de análisis de gestión de costos, con la cual se calcula, a lo largo del ciclo de vida, todos los costos (directos e indirectos) asociados con el desarrollo, operación y el mantenimiento de un proyecto/inversión TI en el modelo tradicional y su comparación ante la implementación en nube pública.
\end{abstract}

Palabras Clave: Modelo Tradicional, Infraestructura como Servicio, Nube Pública, TCO.
Abstract - In Ecuador, the debate has already begun on whether the traditional (on premise) model represents a greater investment in the implementation of IT projects in relation to the public cloud. This study aims to offers an analysis that IT Government administrators should consider when selecting their infrastructure. The Total Cost of Ownership (TCO) model, a cost management analysis tool, is used to calculate, throughout the life cycle, all costs (direct and indirect) associated with development, operation and maintenance of an IT project/investment in the traditional model and its comparison with the public cloud implementation.

Keywords: Traditional Model, Infrastructure as a Service, Public Cloud, TCO

Sumario: I Introducción, II Caso de estudio, III Análisis de resultado, IV Conclusiones

Como citar: Chica, Eduardo. (2020). Análisis de costo total de propiedad (TCO) en un proyecto/inversión TI para el modelo tradicional y su comparación con la implementación en nube pública (IAAS) para las empresas ecuatorianas. Revista Tecnológica - Espol, 32(1). Recuperado a partir de http://www.rte.espol.edu.ec/index.php/tecnologica/article/view/689 


\section{INTRODUCCIÓN}

El rol del departamento de TI ha cambiado. Cada vez es más frecuente solicitar a TI que invierta menos tiempo en la infraestructura, y dedique más tiempo y presupuesto en los servicios de aplicaciones que aumenten valor agregado (ingresos) al negocio. Las empresas se dieron cuenta, que las tecnologías de la información se han convertido en las protagonistas en los últimos años, y esto ha supuesto que el Director de Sistemas debe prepararse para afrontar un cambio en su rol dentro de la empresa para convertirse en un líder. Como consecuencia, el departamento de sistemas adquiere tareas nuevas día a día, y aumenta su responsabilidad, y peso dentro de la organización. En este contexto, se deben lograr dos objetivos fundamentales: saber cuáles son las tecnologías en auge en cada momento y potenciar el negocio adaptándose a cada sector.

Según la Corporación Internacional de Datos (IDC) las previsiones en relación a la transformación digital establecen que, en el año 2020 , el $50 \%$ de los presupuestos dedicados a la división TI estarán enfocados a nuevas iniciativas de transformación digital [3]. Los datos reflejados anteriormente muestran cómo la transformación digital es un proceso que evoluciona muy rápido $\mathrm{y}$ que requiere de líderes para sacar todo su potencial.

Gartner predice que para 2025 el $80 \%$ de las organizaciones a nivel mundial habrán migrado de la infraestructura tradicional a servicios de colocación, alojamiento y nube [2].

En Ecuador según el "Informe del Estado de Adopción de tecnologías de computación en la nube en el Ecuador", indica que el uso de la computación en la nube es aún incipiente; el $42 \%$ de las organizaciones encuestadas tienen menos del $25 \%$ de sus aplicaciones en la nube y un $22 \%$ no la utilizan; la principal barrera para optar por servicios en la nube es la deficiente conexión a Internet que existe en el país. La reducción de costos de infraestructura es la principal motivación que tienen las organizaciones ecuatorianas al momento de optar por servicios en la nube; las pequeñas empresas de tecnología, las universidades, y las ONGs destacan por su uso de servicios en la nube y el $81 \%$ de las organizaciones que no utilizan la nube tienen planes de usarla en el lapso de un año o máximo dos [1]. Según los datos anteriores se debe preparar el terreno para que se rompa el paradigma del modelo tradicional, convirtiendo las oportunidades en cambios hacia un modelo más flexible, seguro y con costos reducidos.

\subsection{Infraestructura tradicional de TI}

A pesar del flujo continuo de mejoras para hardware y software de TI, los desafíos relacionados con la infraestructura que enfrentan los equipos de TI siguen en aumento. El software de infraestructura y virtualización de TI requerido para cubrir las necesidades de las empresas es complejo y costoso, y el manejo de los centros de datos se ha vuelto complicado. Es necesario concentrar mucho tiempo y esfuerzo para mantener todo en funcionamiento.

El modelo on premise (instalado en la localización del usuario) o modelo tradicional presenta limitaciones que impiden a las empresas crecer con la agilidad, y competitividad, a las exigencias del mercado [6], considerando que se debe incurrir en procesos de adquisición e implementación de hardware o software que permitan cubrir una necesidad del negocio [7], lo cual dependiendo de los procesos de compra internos de cada empresa y de la disponibilidad de productos tecnológicos en el mercado local o internacional, por parte de los proveedores, se genera un lapso de tiempo muerto desde que surge la necesidad de los nuevos aplicativos o servicios hasta que se puede iniciar su implementación. Este proceso se debe repetir cada vez que exista una nueva necesidad por parte del negocio y que demande de recursos tecnológicos que la empresa no disponga.

El modelo tradicional está basado en adquirir software y hardware que se amortizará durante los siguientes años. Se trata de un modelo muy sencillo: un integrador de sistemas se encarga de la puesta en marcha y el cliente realiza las tareas administrativas. Un ejemplo claro sería un servidor de correo, en el que el cliente adquiere los servidores, el sistema operativo, las licencias, etcétera y el equipo de TI se encarga de la administración.

La infraestructura tradicional con almacenamiento por separado, redes y servidores como se muestra en la Fig. 1 no es adecuada para cubrir las demandas, cada vez mayores, de las aplicaciones empresariales, ni cubrir el ritmo acelerado de crecimiento de las empresas modernas. Los recursos de almacenamientos creados con la infraestructura tradicional se han convertido, en muchos casos, en la barrera para el cambio y el progreso, aumentando la complejidad, desde el requerimiento de nuevos proyectos, implementación y la administración.

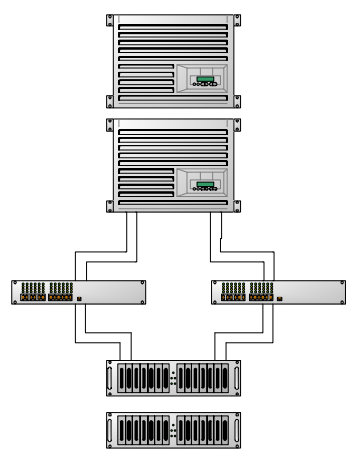

1. COMPLEJIDAD INHERENTE.

2. COMPONENTES INEFICIENTES.

3. ACTUALIZACIONES COSTOSAS.

4. ADMINISTRACIÓN COMPLICADA.

Fig. 1 Desafíos de la infraestructura del modelo tradicional

Las nuevas iniciativas de negocios requieren la participación de varios actores y las necesidades de la organización se deben prever con una anticipación de tres a cinco años. Sin embargo, resulta casi imposible hacerlo en forma correcta, ya que a veces se sobredimensiona o se subdimensiona el recurso de cómputo. Además, los costos en el incremento de la fidelización de los proveedores y de las licencias llevan los presupuestos al límite.

\subsection{Infraestructura de Nube Pública}

La infraestructura de nube está preparada para el uso abierto al público en general. Puede ser de propiedad, administrado y operado por una empresa, academia, organización gubernamental o alguna combinación de ellos [8].

En la actualidad, los equipos de TI empresariales buscan formas de prestar los servicios de TI en sus 
instalaciones con la velocidad, disponibilidad y la eficiencia operativa de los servicios en la nube pública.

En las nubes públicas, los recursos de cómputo como servidores, almacenamiento, software virtualización y demás componentes son propiedad de un proveedor. En este caso, toda esa infraestructura, la ofrece a través de internet o enlaces de datos. Entre las ventajas se puede destacar que es un modelo escalable, no requiere mantenimientos y evita la obsolescencia de equipos, permite una gestión más eficiente en el tiempo de los recursos empresariales, mayor velocidad de implementación y se paga por el uso o la reserva del recurso TI. Entre los proveedores internacionales se puede mencionar: Amazon Web Services (AWS) [9], Microsoft Azure [10], Google Compute Engine [11], IBM [12], y proveedores locales: CNT [13], TELCONET [14], y CLARO [15], cada uno ofrece características, disponibilidad y planes que de forma dinámica se van ajustando a la necesidad de las empresas, por tal es responsabilidad de los administradores de TI evaluar e identificar la mejor opción a la hora de seleccionar un proveedor.

\subsection{TCO (Costo Total de Propiedad)}

Conocido como TCO por sus siglas en inglés (Total Cost of Ownership) es una medida diseñada por el Grupo Gartner para evaluar el costo total en que se incurre al adquirir, inicialmente, un sistema de información y ahora se utiliza su concepto para todas las compras. El TCO es un modelo que ayuda tanto a los consumidores como a los ejecutivos de empresas a evaluar los costos directos e indirectos que están relacionados con la compra de cualquier activo de capital [16].

El principio básico del TCO es que los costos de propiedad de cualquier bien que se adquiere tienen componentes más allá de los estipulados en el precio de compra del mismo, costos en los cuales se debe incurrir para garantizar el funcionamiento correcto del bien o conjunto de bienes, durante la vida útil del mismo. Esta medida surgió por la necesidad de comparar equitativamente propuestas que incluyen valores de cobro permanente al igual que valores de pago único.

El TCO es una visión global de lo que un activo cuesta realmente, más allá del simple coste de adquisición que permita a las empresas o a los consumidores a realizar comparativas precisas entre los distintos productos, servicios o soluciones que estén pensando en adquirir a nivel costes.

El costo total de propiedad de recursos de tecnología incluye los costos originales de adquirir e instalar hardware y software, también los costos por actualizaciones de hardware y software, mantenimiento, soporte técnico, capacitación, e incluso los costos de servicios públicos y la infraestructura física necesarias para la operación de la tecnología.

El modelo de TCO puede ser utilizado para analizar los costos directos e indirectos para ayudar a determinar el costo total real para la implementación de una tecnología específica.

Los costos de adquisición de hardware y software representan solo alrededor del $20 \%$ del TCO [6], así los gerentes deben poner mucha atención sobre los costos administrativos para entender el costo real del hardware y software de la empresa.

A continuación, se muestra un caso de estudio donde se aplica la metodología TCO para comparar los costos totales de un proyecto de tecnología en una infraestructura del modelo tradicional y nube pública.

\section{CASO DE ESTUDIO}

La gerencia de tecnología de la empresa pública Medios Públicos de Comunicación del Ecuador - Medios Públicos EP [17], en su Datacenter de GYE posee la mayoría de sus servidores al término de su vida útil. El tiempo de vida útil promedio de los servidores debería ser de cinco años de acuerdo a las mejores prácticas, y recomendaciones de los fabricantes. El servidor más antiguo tiene 10 años $y$ el más nuevo tiene aproximadamente cinco años de utilización, por lo que actualmente no se cuenta con una infraestructura tecnológica de vanguardia donde se pueda instalar, y garantizar la continuidad de los siguientes sistemas, los mismos que son críticos y vitales para el óptimo funcionamiento de la Empresa. Los sistemas más importantes se muestran en la TABLA I.

TABLA I

SISTEMAS Y SERVICIOS A IMPLEMENTAR POR EL CLIENTE

\begin{tabular}{|l|}
\hline \multicolumn{1}{|c|}{ Descripción del Sistema } \\
\hline Directorio Activo - CUE \\
\hline Antivirus - CUE \\
\hline FileServer - CUE \\
\hline Servidor Contingencia Hermes "Sistema de Distribución" \\
\hline Servidor Contingencia Sistema NAF - Weblogic \\
\hline Servidor Contingencia Sistema NAF -Base Oracle \\
\hline Servidor Archivos Históricos \\
\hline Servidor Sistema Prinergy \\
\hline
\end{tabular}

Los servicios a nivel nacional están operativos las 24 horas, los siete días de la semana. Medios Públicos EP., requiere servidores potentes capaces de soportar y garantizar el acceso a los distintos servicios para todos los usuarios a nivel nacional. Por tal motivo, se requiere adquirir una solución de infraestructura de alta disponibilidad, adaptable, escalable, redundante que permita optimizar los recursos de hardware y donde se contemple la virtualización de los servidores detallados que se muestran en la TABLA II.

TABLA II

RECURSO NECESARIO POR MÁQUINA O SERVIDOR

\begin{tabular}{|c|c|c|c|c|}
\hline Sitio & Servicio & $\begin{array}{c}\text { Almacenamiento } \\
\text { (GB) }\end{array}$ & $\begin{array}{c}\text { Procesamiento } \\
\text { (GB) }\end{array}$ & $\begin{array}{c}\text { Memoria } \\
\text { (GB) }\end{array}$ \\
\hline Cuenca & AD & 180 & 3,4 & 4 \\
\hline Cuenca & Antivirus & 150 & 3,4 & 7 \\
\hline Cuenca & FileServer & 2000 & 3,4 & 4 \\
\hline Matriz & Hemes & 500 & 3,4 & 18 \\
\hline Matriz & NAF & 500 & 3,4 & 18 \\
\hline Matriz & NAF-BD & 600 & 3,4 & 12 \\
\hline Matriz & Archivos & 2000 & 3,4 & 6 \\
\hline Planta & Prinergy & 1200 & 6,8 & 32 \\
\hline & TOTAL & 7130 & 30,6 & 101 \\
\hline
\end{tabular}




\section{A. Costos de la infraestructura de hardware y software del modelo tradicional}

En este caso, es importante asegurar que todos los costos significativos asociados a un sistema informático están considerados en el TCO y cuidar que su contabilización no esté duplicada. Revisar la estructura del TCO permite validar la completitud del cálculo. En el ciclo de vida de un sistema informático, el TCO se descompone en:

- Costos de creación: Costos de selección, diseño, adaptaciones/desarrollo, implantación, formación, gestión del cambio organizativo.

- Costos de operación: Costos de explotación, soporte, mantenimiento correctivo y adaptación evolutiva.

- Costos de reposición o de cierre: Al final de vida útil de un sistema es necesario considerar el costo de migración, actualización o desconexión.

Siguiendo el modelo tradicional, el analista de redes de la institución procedió a solicitar cotizaciones en el mercado que permitan cumplir con el objetivo técnico, considerando como alternativa inicial la compra de equipos de hardware y software para la virtualización de los servidores de la TABLA II en el modelo on premise. La empresa solicitante obtuvo del mercado local la cotización por $\$ 181.104,00$ sin incluir implementación y demás costos antes mencionados.

A continuación, se realiza el análisis TCO para una verdadera evaluación del proyecto y sus alternativas, se considera a los actores que intervienen en el proceso de diseño, implementación, operación y mantenimiento de la nueva infraestructura que soportaría los servicios con la capa de virtualización sin incluir implementación de servicios o aplicaciones de capas superiores.

\section{B. Análisis de TCO}

Con el antecedente anterior, se tiene que considerar la importancia de la visión de un proyecto a lo largo de la vida, desde la creación hasta su sustitución, es necesario reflejar los costos operativos de un sistema y hasta su reemplazamiento. En este sentido se puede afirmar que el TCO depende, en gran manera, de los costos de selección $\mathrm{y}$ diseño del sistema o infraestructura, el esfuerzo dedicado a esta primera etapa definirá el costo y posibilidades de gestión de la etapa de operación.

\section{1) Información del cliente}

La siguiente información es necesaria que se obtenga junto a los siguientes actores: el responsable del departamento de TI y el responsable de talento humano de la empresa.

A continuación, en TABLA III se muestran las fórmulas que se utilizarán en las secciones siguientes para el cálculo de los costos.
TABLA III

\begin{tabular}{|c|c|}
\hline Fórmulas & Descripción \\
\hline (1) & $\begin{array}{l}\text { Salario Anual } \\
=\text { Cantidad de Administradores } \\
*[(\text { Salario Mensual } \\
*(1+\% \text { Aporte patronal al IESS })) * 12 \\
+ \text { Decimo TercerSueldo + Decimo Cuarto Sueldo }]\end{array}$ \\
\hline (2) & $\begin{array}{l}\text { Costo Hora Admin } \\
=((\text { Salario Anual } / 52 \text { semanas }) / 40 \text { horas }) \\
* \text { Porcentaje tiempo Administrador }\end{array}$ \\
\hline (3) & $\begin{array}{l}\text { Costo adm de plataforma primer año } \\
=\text { Costo hora Admin } * \text { (Horas Instalación } \\
\text { + Horas Mantenimiento } \\
\text { + Horas Especialización) } \\
\text { * Cantidad de nodos }\end{array}$ \\
\hline (4) & $\begin{array}{l}\text { Costo Electricidad primer año } \\
\qquad \begin{array}{l}=\text { Costo del Kw } \\
\text { /h según arconel } \\
\\
* \text { Consumo Kw } \\
\\
\text { /h por nodo } \\
\\
* \text { Cantidad de nodos } \\
* \text { Horas por año }\end{array}\end{array}$ \\
\hline (5) & $\begin{array}{l}\text { Costo Anual Inf raestrucutura } \\
\text { = Costo Aire Acondicionado + (Costo Oficina } \\
* 12) \text { + (Pago Mensual Enlace de Internet } * 12) \\
\text { + Pago de Inscripción Enlace de Internet } \\
\text { + (Pago Mensual Enlace de Datos } * 12) \\
\text { + Pago de Inscripción Enlace de Datos } \\
\text { + (Pago Electricidad/2) }\end{array}$ \\
\hline (6) & $\begin{array}{l}\text { Costo Soluciñon Primer Año } \\
\text { = PAGO(Porcentaje Costo de Oportunidad; (Meses } \\
\text { Amortización/12); - (Costo Licenciamiento + } \\
\text { Costo por Administración Plataforma + } \\
\text { Costo Respaldo + Costo Electricidad + } \\
\text { Costo Servidores + } \\
\text { Costo Componentes Infraestructura + } \\
\text { Costo Implementación de Infraestructura + } \\
\text { Costo Infraestructura) * } \\
\text { Meses de Amorticazión/12 }\end{array}$ \\
\hline (7) & $\begin{array}{l}\text { Costo Recurrente Mantenimiento Hardware } \\
=0,05 * \text { Costo Servidores }\end{array}$ \\
\hline (8) & $\begin{array}{l}\text { Costo Recurrente Administración } \\
=(\text { Cantidad de horas de Mantenimiento Hardware } \\
\text { + Cantidad Horas Mantenimiento Software }) \\
\text { * Costo hora Administrador } * \text { Número de nodos }\end{array}$ \\
\hline (9) & $\begin{array}{l}\text { Costo Recurrente Mantenimiento } \\
\text { Infraestructura } \\
=(\text { PM Enlace Datos } * 12) \\
+(\text { PM Enlace de Internet }=12) \\
+(\text { Costo Oficina } * 12) \\
+(\text { Costo Electricidad de Infraestructura })\end{array}$ \\
\hline (10) & $\begin{array}{l}\text { Costo Total Recurrente } \\
\text { = Mantenimiento de licenciamiento } \\
\text { + Costo Mantemiento de hardware } \\
\text { + Costo Electricidad + Costo Administración } \\
\text { + Costo Mantenimiento Infraestructura }\end{array}$ \\
\hline
\end{tabular}


A continuación, en la TABLA IV se describen las variables más importantes a considerar en el caso de estudio.

TABLA IV

INFORMACIÓN DE LA INSTITUCIÓN

\begin{tabular}{|c|c|c|c|}
\hline Variables Globales & Cantidad & Descripción & Fórmulas \\
\hline $\begin{array}{l}\text { Cantidad de } \\
\text { Administradores de } \\
\text { Infraestructura } \\
\end{array}$ & 2 & $\begin{array}{l}\text { Información de los } \\
\text { administradores de } \\
\text { la infraestructura } \\
\end{array}$ & \\
\hline $\begin{array}{l}\text { Porcentaje de Tiempo } \\
\text { dedicado a administrar } \\
\text { la infraestructura }\end{array}$ & 0,5 & $\begin{array}{l}\text { Información de los } \\
\text { administradores de } \\
\text { la infraestructura }\end{array}$ & \\
\hline $\begin{array}{l}\text { Salario mensual } \\
\text { Administrador de } \\
\text { Infraestructura }\end{array}$ & $\begin{array}{c}\$ \\
1.100,00\end{array}$ & $\begin{array}{l}\text { Ingrese el salario } \\
\text { mensual del } \\
\text { administrador de } \\
\text { infraestructura }\end{array}$ & $\begin{array}{l}\text { Ver } \\
\text { TABLA } \\
\text { III. } \\
\text { Fórmula } \\
\# 1 \\
\end{array}$ \\
\hline $\begin{array}{l}\text { Salario anual } \\
\text { Administrador de } \\
\text { Infraestructura }\end{array}$ & $\begin{array}{c}\$ \\
32.293,60\end{array}$ & $\begin{array}{l}\text { Se calcula } \\
\text { automáticamente } \\
\text { considerando el } \\
\text { salario ingresado } \\
\text { más los beneficios } \\
\text { de ley y } \\
\text { aportaciones al } \\
\text { seguro social } \\
\end{array}$ & \\
\hline $\begin{array}{l}\text { Costo por hora de } \\
\text { Administrador de } \\
\text { Infraestructura }\end{array}$ & $\begin{array}{c}\$ \\
7,76\end{array}$ & $\begin{array}{l}\text { Costo por hora del } \\
\text { administrador de } \\
\text { infraestructura } \\
\text { considerando } \\
\text { aportes a la } \\
\text { seguridad social y } \\
\text { demás beneficios } \\
\end{array}$ & $\begin{array}{l}\text { Ver } \\
\text { TABLA } \\
\text { III. } \\
\text { Fórmula } \\
\# 2\end{array}$ \\
\hline Meses de amortización & 36 & $\begin{array}{l}\text { Amortización/ } \\
\text { Depreciación } \\
\text { activos de hardware }\end{array}$ & \\
\hline $\begin{array}{l}\text { Instalación de un nodo } \\
(\mathrm{HW}+\mathrm{SW})\end{array}$ & 30 & $\begin{array}{l}\text { Horas de } \\
\text { instalación de un } \\
\text { servidor con } \\
\text { sistema operativo } \\
\text { base, networking, } \\
\text { seguridad } \\
\end{array}$ & \\
\hline $\begin{array}{l}\text { Especialización del } \\
\text { nodo }\end{array}$ & 60 & $\begin{array}{l}\text { Horas para instalar } \\
\text { y configurar la capa } \\
\text { de aplicación }\end{array}$ & \\
\hline $\begin{array}{l}\text { Mantenimiento y } \\
\text { soporte para el nodo }\end{array}$ & 60 & $\begin{array}{l}\text { Horas de soporte y } \\
\text { mantenimiento del } \\
\text { servidor en el } \\
\text { primer año }\end{array}$ & \\
\hline $\begin{array}{l}\text { Costo por TB de } \\
\text { Respaldo }\end{array}$ & $-\$$ & $\begin{array}{l}\text { Cubre tiempo de } \\
\text { administración, } \\
\text { cintas, espacio en } \\
\text { disco y licencias de } \\
\text { software, basado en } \\
\text { información } \\
\text { proporcionada por } \\
\text { la Gerencia de Data } \\
\text { Center y tomando } \\
\text { como consideración } \\
\text { la plataforma } \\
\text { utilizada para } \\
\text { respaldo de } \\
\text { información }\end{array}$ & \\
\hline $\begin{array}{l}\text { Mantenimiento del } \\
\text { Software }\end{array}$ & 40 & $\begin{array}{l}\text { Número de horas } \\
\text { de mantenimiento } \\
\text { del software por } \\
\text { año }\end{array}$ & \\
\hline $\begin{array}{l}\text { Mantenimiento del } \\
\text { Hardware }\end{array}$ & 60 & $\begin{array}{l}\text { Número de horas } \\
\text { de mantenimiento } \\
\text { del hardware por } \\
\text { año }\end{array}$ & \\
\hline
\end{tabular}

2) Costos Primer Año en el Modelo Tradicional

En la TABLA V, se encuentra que el costo total para el primer año en el modelo tradicional es $\$ 288.096,35$ se consideran los costos de administración, electricidad, aire acondicionado, servidores, componentes de red, implementación y costo de oportunidad:

TABLA V

COSTOS PRIMER AÑO MODELO TRADICIONAL

\begin{tabular}{|c|c|c|c|}
\hline $\begin{array}{c}\text { Variables } \\
\text { Globales }\end{array}$ & Cantidad & Descripción & Formulas \\
\hline $\begin{array}{l}\text { Costo de } \\
\text { Licenciamiento } \\
\text { por Nodo: }\end{array}$ & $\$$ & $\begin{array}{l}\text { Licencias de } \\
\text { Virtualización } \\
\text { (En este caso es } \\
\$ 0 \text { debido que el } \\
\text { virtualizador viene } \\
\text { incluido en el } \\
\text { hardware) }\end{array}$ & \\
\hline $\begin{array}{l}\text { Licenciamiento } \\
\text { de Nodos: }\end{array}$ & $\$$ & $\begin{array}{l}\text { Licenciamiento de } \\
\text { virtualización para } \\
\text { servidores (En } \\
\text { este caso es } \$ 0 \\
\text { debido que el } \\
\text { virtualizador viene } \\
\text { incluido en el } \\
\text { hardware) }\end{array}$ & \\
\hline $\begin{array}{l}\text { Costos por } \\
\text { tiempo de } \\
\text { administración } \\
\text { de plataformas: }\end{array}$ & $\$ \quad 3.493,30$ & & $\begin{array}{l}\text { Ver } \\
\text { TABLA } \\
\text { III. } \\
\text { Fórmula } \\
\text { \#3 }\end{array}$ \\
\hline $\begin{array}{l}\text { Costos de } \\
\text { Respaldo: }\end{array}$ & $\$$ & & \\
\hline Electricidad: & $2.099,52$ & $\begin{array}{l}\text { Tarifas del Sector } \\
\text { Eléctrico - } \\
\text { ARCONEL }\end{array}$ & $\begin{array}{l}\text { Ver } \\
\text { TABLA } \\
\text { III. } \\
\text { Fórmula } \\
\quad \# 4 \\
\end{array}$ \\
\hline Servidores: & $\$ 135.000,00$ & & \\
\hline $\begin{array}{l}\text { Componentes } \\
\text { Infraestructura: }\end{array}$ & $\$ 11.700,00$ & & \\
\hline $\begin{array}{l}\text { Implementación } \\
\text { Infraestructura: }\end{array}$ & $\$ 17.000,00$ & & \\
\hline $\begin{array}{l}\text { Pago Mensual } \\
\text { Enlace } \\
\text { Comunicación: }\end{array}$ & $\$$ & $\begin{array}{l}\text { Enlace de datos } \\
\text { desde el } \\
\text { datacenter a la } \\
\text { oficina principal }\end{array}$ & \\
\hline $\begin{array}{l}\text { Pago Inscripción } \\
\text { Enlace } \\
\text { Comunicación: }\end{array}$ & $\$$ & $\begin{array}{l}\text { Enlace de datos } \\
\text { desde el } \\
\text { datacenter a la } \\
\text { oficina principal } \\
\text { inscripción }\end{array}$ & \\
\hline $\begin{array}{l}\text { Pago Mensual } \\
\text { Enlace Internet: }\end{array}$ & $\$$ & $\begin{array}{l}\text { Enlace de internet } \\
\text { datacenter }\end{array}$ & \\
\hline $\begin{array}{l}\text { Pago Inscripción } \\
\text { Enlace Internet }\end{array}$ & $\$$ & $\begin{array}{l}\text { Enlace de internet } \\
\text { datacenter } \\
\text { inscripción }\end{array}$ & \\
\hline $\begin{array}{l}\text { Precio Aire } \\
\text { Acondicionado: }\end{array}$ & 350,00 & $\begin{array}{l}\text { Valores } \\
\text { proporcionados } \\
\text { por el cliente }\end{array}$ & \\
\hline Costo Oficinas: & $\$ \quad 5.000,00$ & $\begin{array}{l}\text { Valores } \\
\text { proporcionados } \\
\text { por el cliente }\end{array}$ & \\
\hline Infraestructura: & $\$ 61.399,76$ & $\begin{array}{l}\text { Redes de } \\
\text { Comunicación } \\
\text { (datos y } \\
\text { comunicación), } \\
\text { oficinas, Internet, } \\
\text { aire } \\
\text { acondicionado y } \\
\text { ventilación }\end{array}$ & $\begin{array}{l}\text { Ver } \\
\text { TABLA } \\
\text { III. } \\
\text { Fórmula } \\
\quad \# 5\end{array}$ \\
\hline $\begin{array}{l}\text { Costo de } \\
\text { oportunidad: }\end{array}$ & $11,99 \%$ & $\begin{array}{l}\text { Interés anual de } \\
\text { los bancos en caso } \\
\text { de inversión }\end{array}$ & \\
\hline Total: & \$ 288.096,35 & & $\begin{array}{c}\text { Ver } \\
\text { TABLA } \\
\text { III. } \\
\text { Fórmula } \\
\quad \# 6 \\
\end{array}$ \\
\hline
\end{tabular}


3) Costos recurrentes en el Modelo Tradicional

Los costos recurrentes luego del primer año son \$ $72.228,15$ (costos electricidad, administración, costos de oficina, y mantenimiento de la infraestructura). En la TABLA VI se puede verificar estos costos.

TABLA VI

COSTOS RECURRENTES ANUALES EN EL MODELO TRADICIONAL

\begin{tabular}{|c|c|c|c|}
\hline Variables Globales & Cantidad & Descripción & Formulas \\
\hline $\begin{array}{l}\text { Mantenimiento de } \\
\text { licenciamiento: }\end{array}$ & $\$$ & $\begin{array}{l}20 \% \text { de costos de } \\
\text { licenciamiento } \\
\text { por } \\
\text { mantenimiento } \\
\text { anual (software, } \\
\text { almacenamiento } \\
\text { y respaldo) }\end{array}$ & $\begin{array}{l}\text { En este } \\
\text { caso es } \$ 0 \\
\text { debido que } \\
\text { el } \\
\text { virtualizado } \\
\text { viene } \\
\text { incluido en } \\
\text { el hardware }\end{array}$ \\
\hline $\begin{array}{l}\text { Contrato de } \\
\text { servicio de } \\
\text { mantenimiento de } \\
\text { Hardware: }\end{array}$ & $\$ 6.750,00$ & $\begin{array}{l}5 \% \text { anual del } \\
\text { costo total de } \\
\text { servidores }\end{array}$ & $\begin{array}{l}\text { Ver } \\
\text { TABLA } \\
\text { III. } \\
\text { Fórmula } \\
\# 7 \\
\end{array}$ \\
\hline Electricidad: & $\$ 2.099,52$ & & \\
\hline $\begin{array}{l}\text { Costos de } \\
\text { administración: }\end{array}$ & $\$ 2.328,87$ & & $\begin{array}{l}\text { Ver } \\
\text { TABLA } \\
\text { III. } \\
\text { Fórmula } \\
\# 8\end{array}$ \\
\hline $\begin{array}{l}\text { Pago Mensual } \\
\text { Enlace } \\
\text { Comunicación: }\end{array}$ & $\$$ & Enlace de Datos & \\
\hline $\begin{array}{l}\text { Pago Mensual } \\
\text { Enlace Internet: }\end{array}$ & $\$$ & $\begin{array}{l}\text { Enlace de } \\
\text { Internet }\end{array}$ & \\
\hline Costo Oficinas: & $\$ 5.000,00$ & & \\
\hline $\begin{array}{l}\text { Mantenimiento de } \\
\text { Infraestructura: }\end{array}$ & $\$ 61.049,76$ & $\begin{array}{l}\text { Redes de } \\
\text { Comunicación, } \\
\text { aire } \\
\text { acondicionado y } \\
\text { ventilación, } \\
\text { oficinas, Internet }\end{array}$ & $\begin{array}{l}\text { Ver } \\
\text { TABLA } \\
\text { III. } \\
\text { Fórmula } \\
\# 9\end{array}$ \\
\hline Total: & $\$ 72.228,15$ & & $\begin{array}{l}\text { Ver } \\
\text { TABLA } \\
\text { III. } \\
\text { Fórmula } \\
\# 10\end{array}$ \\
\hline
\end{tabular}

4) Sub-cálculos

Al pie el detalle en la TABLA VII los sub-cálculos realizados para obtener las matrices antes indicadas.

TABLA VII

VALORES PARA EL SUB-CÁLCULOS

\begin{tabular}{|c|c|c|c|}
\hline $\begin{array}{c}\text { Variables } \\
\text { adicionales }\end{array}$ & \multicolumn{2}{|c|}{ Cantidad } & Descripción \\
\hline $\mathbf{k w} / \mathbf{h}$ & $\$$ & 0,08 & $\begin{array}{c}\text { Tarifa comercial según } \\
\text { Arconel }\end{array}$ \\
\hline $\begin{array}{r}\text { Número de horas en } \\
1 \text { año }\end{array}$ & & 8640 & \\
\hline $\begin{array}{r}\text { Consumo promedio } \\
\text { kw/h por nodo }\end{array}$ & & 1 & $\begin{array}{l}\text { Documentación pública } \\
\text { del fabricante } \\
\text { NUTANIX } \\
\text { (https://www.nutanix.co } \\
\text { m/products/hardware- } \\
\text { platforms/) }\end{array}$ \\
\hline $\begin{array}{r}\text { Total espacio } \\
\text { requerido de } \\
\text { almacenamiento }\end{array}$ & & 7,11 & $\begin{array}{l}\text { Capacidad en Terabytes } \\
\text { de almacenamiento }\end{array}$ \\
\hline $\begin{array}{r}\text { Valor Referencial } \\
\text { Unitario de Cada } \\
\text { Nodo }\end{array}$ & $\$$ & $45.000,00$ & \\
\hline Total Hardware & $\$$ & $135.000,00$ & $\begin{array}{c}\text { Six } 10 \text { cores }(2,4 \mathrm{GHz}, \\
288 \mathrm{~GB} \text { RAM })\end{array}$ \\
\hline
\end{tabular}

\begin{tabular}{|c|c|c|}
\hline $\begin{array}{r}\text { Servidores } \\
\text { necesarios }\end{array}$ & 3 & $\begin{array}{c}\text { Basado en el número de } \\
\text { cores construidos con } \\
\mathrm{N}+1 \text { para } 96 \% \text { y }+2 \\
\text { para } 98 \%\end{array}$ \\
\hline
\end{tabular}

5) Costos unitarios en el Modelo Cloud (Proveedor Local CNT)

En la TABLA VIII, se muestran los valores unitarios por la renta de recurso (procesamiento, memoria, almacenamiento y monitoreo) con estos valores se obtiene la renta mensual del recurso del cliente en el modelo de nube pública.

TABLA VIII

Costos Unitarios Considerados PARA LOS SERVICIO CLOUD

\begin{tabular}{|c|c|c|c|}
\hline \multicolumn{4}{|c|}{ Recursos Cloud } \\
\hline Variables & & tario & Notas \\
\hline Procesamiento & $\$$ & 8,18 & Valor por Core \\
\hline Memoria & $\$$ & 8,67 & Valor por GB Memoria \\
\hline Almacenamiento & $\$$ & 0,47 & $\begin{array}{l}\text { Valor por GB } \\
\text { Almacenamiento }\end{array}$ \\
\hline Monitoreo & $\$$ & 50,00 & $\begin{array}{l}\text { Valor de Monitoreo DCV } \\
\text { (pago mensual) }\end{array}$ \\
\hline
\end{tabular}

\section{ANÁLISIS DE RESULTADO}

Luego de obtener el costo total del primer año en el modelo tradicional y el costo promedio mensual de la solución de nube pública, se puede indicar en la TABLA IX, que el ahorro promedio estimado mensual entre la solución nube pública vs modelo tradicional es de $64 \%$ en el primer mes.

TABLA IX

RESULTADOS DEL MODELO CLOUD VS MODELO TRADICIONAL EN EL PRIMER MES

\begin{tabular}{|c|c|c|}
\hline \multicolumn{3}{|l|}{ Resultados } \\
\hline Número de CPU: & & 12 \\
\hline Memoria (GB): & & 01 \\
\hline Almacenamiento Gold (GB): & & 7130 \\
\hline Monitoreo: & & 1 \\
\hline $\begin{array}{r}\text { Disponibilidad de la solución modelo } \\
\text { tradicional: }\end{array}$ & & $96,0 \%$ \\
\hline $\begin{array}{r}\text { Amortización / Depreciación activos de } \\
\text { hardware (Meses): }\end{array}$ & & 36 \\
\hline $\begin{array}{r}\text { Costo promedio mensual de la solución } \\
\text { modelo tradicional: }\end{array}$ & $\$$ & $12.015,35$ \\
\hline Costo promedio solución Cloud: & $\$$ & $4.288,23$ \\
\hline Promedio estimado mensual de ahorros: & & $64 \%$ \\
\hline
\end{tabular}

En la Fig. 2, se logra observar con mayor detalle el ahorro de inversión desde el primer año. Se evidencian los costos de reposición o cierre en cada ciclo de la TABLA VI que es obligatorio en el modelo tradicional a diferencia del modelo nube pública. 
Comparación global de costos

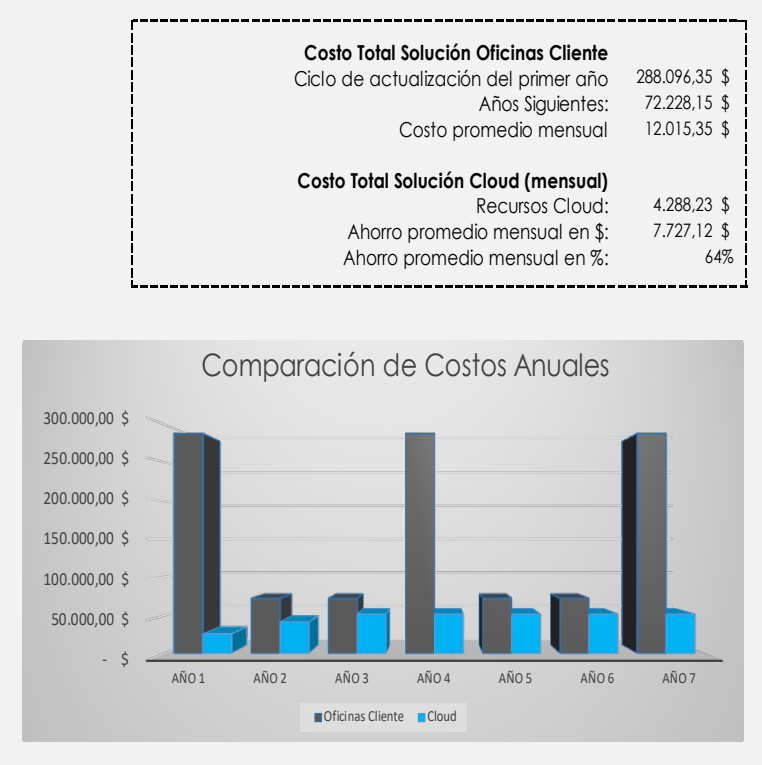

Fig. 2 Comparación de los costos anuales

Finalmente, en la Fig. 3 se observa que el ahorro de la solución a tres años es de un $73 \%$ entre la solución Cloud vs modelo tradicional.

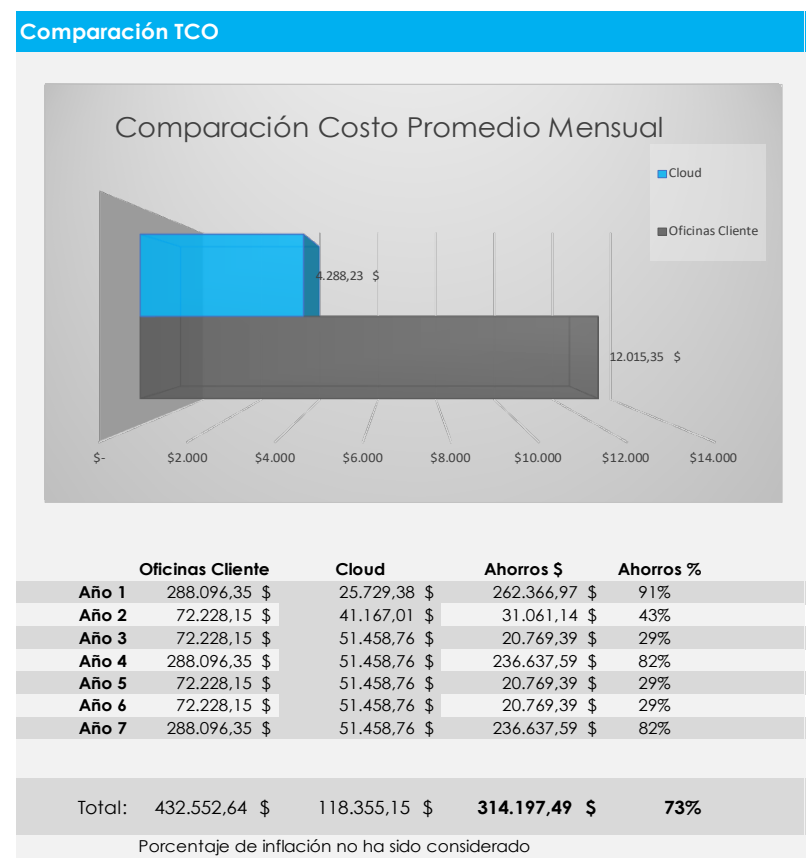

Fig. 3 Comparación de Costo Promedio Mensual y Costos por año

\section{CONCLUSIONES}

La nube pública se ha convertido en la base que permite a las empresas obtener una ventaja competitiva en comparación a aquellas que siguen el modelo tradicional. Las estrategias de la nube están evolucionando rápidamente hacia la analítica y al Internet de las cosas (IOT). En este contexto, las organizaciones deben seguir invirtiendo y madurando su competencia en la nube para asegurarse que se convierta en la plataforma principal de la organización. La nube se encuentra asociada a las características básicas, reducción de costos, tiempos y su potencial para transformar los modelos de negocios. Las empresas que quieran sobrevivir a la actual revolución digital van a tener que desmaterializarse, mantener una infraestructura física ya no es rentable. La nube pública se presenta como una plataforma tecnológica emergente que proporciona importantes capacidades a las empresas para centrarse en los objetivos planificados, lo que les permite acelerar sus productos al mercado con una gran libertad económica, con el fin de responder a los cambios del mercado y la demanda de sus clientes.

La herramienta Coste Total de Propiedad es una metodología de análisis para considerar todos los componentes de costos a lo largo de la vida útil de un sistema informático, es especialmente válido para aflorar aquellos costos adicionales a la adquisición de equipos y software que pueden no ser evidentes llamados también costos ocultos. Según los resultados del caso de estudio el cliente llegaría ahorrarse hasta un 64\% mensual, frente al modelo tradicional. Y si se realiza el análisis a tres años se ahorraría hasta un $73 \%$, si trabaja en un modelo de nube pública.

Finalmente, en la siguiente TABLA $X$ se puede observar las características adicionales que reafirman que el modelo de nube pública es mucho más conveniente a la hora de decidir por la compra de infraestructura para el modelo tradicional en flexibilidad, costos, disponibilidad, soporte, servicios, características técnicas, seguridad y tiempos de implementación.

TABLA X

COMPARATIVA DEL MODELO EN NUBE VS TRADICIONAL

\begin{tabular}{|r|c|c|}
\hline \multicolumn{2}{|c|}{ Nube Pública vs. Modelo Tradicional } \\
\hline Característica & Tradicional & Nube \\
\hline $\begin{array}{r}\text { Disponibilidad Nube Pública } \\
\text { Local }\end{array}$ & mínima & alta \\
\hline $\begin{array}{r}\text { Amortización / Depreciación } \\
\text { activos de hardware }\end{array}$ & $36,0 \%$ & $99,98 \%$ \\
\hline Soporte 24 X 7 & NO & N/A \\
\hline $\begin{array}{r}\text { Servicio de Migración } \\
\text { datos de 60000 IOPS }\end{array}$ & NO & SI \\
\hline $\begin{array}{r}\text { Velocidad de lectura/escritura de } \\
\text { Fuentes de poder redundantes }\end{array}$ & NO & SI \\
\hline $\begin{array}{r}\text { Costos RRHH para operación } \\
\text { Seguridad en la red de } \\
\text { comunicaciones }\end{array}$ & Adicional & Incluido \\
\hline Escalabilidad & NO & Incluido \\
\hline Tiempo de Implementación & 3 meses aprox. & 1 mes \\
\hline
\end{tabular}

\section{REFERENCIAS}

[1] C. Abad, M. Villavicencio, E. Boza, and G. Ortega, "Informe del estado de adopción de tecnologías de computación en la nube en el ecuador", Escuela Superior Politécnica del Litoral, Guayaquil, 2017.

[2] (2017) The Gartner website. [Online]. Available: http://www.gartner.com/newsroom/id/3616417

[3] (2018) The IDC website. [Online]. Available: https://mba.americaeconomia.com/articulos/notas/reporte-idc-para2022-se-digitalizara-mas-del-50-del-pib-de-america-latina. Americaeconomia

[4] El Comercio, "La Educación Ecuatoriana se apoya en la nube", pp.4, 2018.

[5] (2017) The Nutanix website. [Online]. Available: https://innovadessstec.com/2019/04/02/la-guia-definitiva-parainfraestructura-hiperconvergente/

[6] K. Laudon, and J. Laudon, "Management Information Systems", 12th ed., New York, USA: Prentice Hall, 2011. 
[7] G. Reese, "Cloud Application Architectures", 1a. Ed., Cambridge, USA, O'Reilly Media, 2009.

[8] (2011) The NIST website. [Online]. Available: http://csrc.nist.gov/publications/nistpubs/800-145/SP800-145.pdf

[9] (2020) The Amazon Web Services (AWS) website. [Online]. Available: https://calculator.s3.amazonaws.com/index.html

[10] (2020) The Microsoft Azure website. [Online]. Available: https://azure.microsoft.com/en-in/pricing/calculator/

[11] (2020) The Google Compute Engine website. [Online]. Available: https://cloud.google.com/products/calculator/?hl=es-419

[12] (2020) The IBM website. [Online]. Available: https://www.ibm.com/cloud/pricing

[13] (2020) La Corporación Nacional de Telecomunicaciones website. [Online]. Available:

https://www.cntempresas.com/soluciones/infraestructura/datacenter-virtual/

[14] (2020) Telconet S.A. website. [Online]. Available: https://www.telconet.net/index.php/soluciones/cloud/cloud-iaas

[15] (2020) Claro website. [Online]. Available: https://www.clarocloud.com.ec/portal/ec/cld/infraestructura/data_c enter virtual/\#info-02

[16] L. Mierutz and B. Kirwin, "Defining Gartner Total Cost of Ownership", Gartner, G00131837,2005.

[17] Perfil de Proyecto Adquisición de servidores para proyectos multipropósitos, Medios Públicos E.P., 2018. 